\title{
Plane-wave pseudopotential implementation of explicit integrators for time-dependent Kohn-Sham equations in large-scale simulations
}

\author{
André Schleife, ${ }^{1}$ Erik W. Draeger, ${ }^{2}$ Yosuke Kanai, ${ }^{1,3, a)}$ and Alfredo A. Correa ${ }^{1, a)}$ \\ ${ }^{1}$ Condensed Matter and Materials Division, Lawrence Livermore National Laboratory, Livermore, \\ California 94550, USA \\ ${ }^{2}$ Center for Applied Scientific Computing, Lawrence Livermore National Laboratory, Livermore, \\ California 94550, USA \\ ${ }^{3}$ Department of Chemistry, The University of North Carolina at Chapel Hill, Chapel Hill, \\ North Carolina 27599-3290, USA
}

(Received 30 July 2012; accepted 28 September 2012; published online 22 October 2012)

\begin{abstract}
Explicit integrators for real-time propagation of time-dependent Kohn-Sham equations are compared regarding their suitability for performing large-scale simulations. Four algorithms are implemented and assessed for both stability and accuracy within a plane-wave pseudopotential framework, employing the adiabatic approximation to the exchange-correlation functional. Simulation results for a single sodium atom and a sodium atom embedded in bulk magnesium oxide are discussed. While the first-order Euler scheme and the second-order finite-difference scheme are unstable, the fourthorder Runge-Kutta scheme is found to be conditionally stable and accurate within this framework. Excellent parallel scalability of the algorithm up to more than a thousand processors is demonstrated for a system containing hundreds of electrons, evidencing the suitability for large-scale simulations based on real-time propagation of time-dependent Kohn-Sham equations. (C) 2012 American Institute of Physics. [http://dx.doi.org/10.1063/1.4758792]
\end{abstract}

\section{INTRODUCTION}

Accurately describing the quantum dynamics of electrons in numerical simulations is crucial for addressing a number of important problems in materials physics as well as chemical physics. For example, detailed understanding of the electron transfer mechanism across material interfaces is important for improving photovoltaic cells ${ }^{1}$ or massive electron excitation in materials under matter radiation. ${ }^{2}$ While analytical models often work reliably for describing electron transport phenomena in simple and homogeneous systems (such as organic molecules or bulk solids), quantitative simulations are necessary to accurately describe the dynamical effects of electrons in complex environments. The proper treatment of the electron dynamics is also necessary for describing highly nonequilibrium many-body electron-ion processes such as the radiation damages in materials. A variety of numerical simulations, semi-empirical ${ }^{3}$ and $a b$ initio, however, lack the essential component: the response of the electrons to a large perturbation of the atomic motion. This is because the aforementioned approaches rely on the adiabatic BornOppenheimer (BO) approximation, ${ }^{4}$ i.e., on the assumption that the electrons adjust instantaneously to moving ions (e.g., by remaining in the ground state). This approximation can be overcome, for instance, by Ehrenfest dynamics ${ }^{5}$ or the surface-hopping approach for a more sophisticated treatment of the electron dynamics. ${ }^{6}$

Time-dependent density functional theory (TDDFT) ${ }^{7}$ is an attractive approach for describing quantum dynamics of electrons in materials because of its well-balanced accuracy

\footnotetext{
a) Authors to whom correspondence should be addressed. Electronic addresses: ykanai@unc.edu and correaa@1lnl.gov.
}

and efficiency. TDDFT, as a formal extension of groundstate $\mathrm{DFT}^{8}$ for the treatment of time-dependent Hamiltonians, has been applied to various problems in many different areas ranging from materials science to biochemistry (see, e.g., Ref. 9 and references therein). The popularity of TDDFT in various fields has led to a number of recent developments in the formal theory itself as well as in the practical aspect of implementing the theory for numerical calculations. ${ }^{10-14}$

While the majority of applications currently exploits the linear-response formulation of TDDFT ${ }^{15}$ to investigate the excitation of electrons based on the ground-state solution of the Kohn-Sham (KS) equations, ${ }^{16}$ the so-called real-time TDDFT (RT-TDDFT) aims at explicitly obtaining the timedependence of electronic states through the time-dependent KS Hamiltonian. Using RT-TDDFT, it is possible to compute electronic excitation spectra from the dynamics itself, in addition to investigating the quantum dynamics of electrons in real time. The RT-TDDFT approach is gaining increasing popularity as many time-dependent phenomena are becoming an important focus of modern materials research.

In this work, we numerically assess four explicit integrators that have been successfully and widely used to solve ordinary differential equations, regarding their suitability for the time-dependent Kohn-Sham (TDKS) equationsa set of coupled and nonlinear partial differential equations. The integrators that we employ belong to the category of explicit (time forward), spectral (spatial plane-wave basis set) methods and feature an appealing algorithmic suitability for large-scale simulations. We have built our RT-TDDFT implementation upon the Qbox code, ${ }^{17,18}$ a highly scalable DFT code based on the plane-wave pseudopotential formalism. The excellent scalability of underlying components in 
the code and employing explicit integrators allows us to immediately apply the new method to the large heterogeneous systems needed to explore modern applications. At the same time, the plane-wave basis will ensure broad applicability to a diverse set of materials and even periodic bulk solids, as numerical convergence can be rigorously tested by systematically increasing the basis size. We stress that the interplay of accuracy and numerical stability observed in this work is not obvious and cannot be expected a priori when classical integration methods are adapted for the nonlinear TDKS partial differential equations.

The paper is structured as follows: In Sec. II, the theoretical framework is outlined and computational details are discussed. In particular, the conservation of energy is presented as a criterion to assess the numerical stability and accuracy of the propagators that are introduced in Sec. III. In the following, these integrators are applied to two test systems, that represent two limiting cases: (i) an isolated sodium (Na) atom (Sec. IV) and (ii) a periodic solid, modeled using a 64-atom supercell containing a $\mathrm{Na}$ atom embedded in bulk magnesium oxide ( $\mathrm{MgO})(\mathrm{Sec} . \mathrm{V})$. Finally, Sec. VI summarizes the findings of this work.

\section{THEORETICAL FRAMEWORK AND NUMERICAL TREATMENT}

\section{A. Time-dependent Kohn-Sham equations}

TDDFT is based on the one-to-one correspondence between the (time-dependent) one-particle density $n(\mathbf{r}, t)$ and the (time-dependent) one-particle potential $V_{\text {ext }}(\mathbf{r}, t)$ acting on a fictitious system of non-interacting particles with the (same) ground-state density. This correspondence is established by the Runge-Gross theorem ${ }^{7}$ which formally extends the Hohenberg-Kohn theorem ${ }^{8}$ to the time-dependent case. As a consequence of the Runge-Gross theorem, it is possible to also generalize the fictitious system of non-interacting KS particles ${ }^{16}$ under the influence of an effective KS potential to the time-dependent case. These TDKS equations read

$$
\begin{aligned}
\mathrm{i} \hbar \frac{\mathrm{d}}{\mathrm{d} t}\left|\phi_{i}(t)\right\rangle & =\hat{H}(t)[n]\left|\phi_{i}(t)\right\rangle \\
& =\left\{\hat{T}+\hat{V}_{\mathrm{ext}}(t)+\hat{V}_{\mathrm{HXC}}[n]\right\}\left|\phi_{i}(t)\right\rangle .
\end{aligned}
$$

In Eq. (1), $\hat{T}$ is the kinetic energy operator $-\frac{\hbar^{2}}{2 m_{\mathrm{e}}} \nabla_{\mathbf{r}}^{2}$ and $V_{\mathrm{HXC}}[n](\mathbf{r}, t)=\int \frac{n\left(\mathbf{r}^{\prime}, t\right)}{\left|\mathbf{r}-\mathbf{r}^{\prime}\right|} \mathrm{d} \mathbf{r}^{\prime}+\frac{\delta E_{\mathrm{XC}}}{\delta n(\mathbf{r}, t)}$ is the sum of the Hartree $(\mathrm{H})$ potential and the exchange-correlation $(\mathrm{XC})$ potential, which is derived from a universal XC functional $E_{\mathrm{XC}}[n]$. The electron density $n$ follows from the occupied KS orbitals (labeled by the index $i$ ) according to

$$
n(\mathbf{r}, t)=\sum_{i}\left|\phi_{i}(\mathbf{r}, t)\right|^{2} .
$$

RT-TDDFT aims at obtaining the (time-dependent) solutions to the nonlinear TDKS equations (1) for given initial conditions for $\phi_{i}$. It is assumed that the so-called adiabatic functional approximation ${ }^{19}$ (in which the XC functional depends only on the electron density at instantaneous time) is fulfilled, i.e., $V_{\mathrm{HXC}}[n](\mathbf{r}, t) \equiv V_{\mathrm{HXC}}^{\mathrm{adiab}}[n(t)](\mathbf{r})=\frac{\partial E_{\mathrm{HXC}}}{\partial n(\mathbf{r})}[n(t)]$. In addition, the external potentials $V_{\text {ext }}$ studied in this work are not explicitly time dependent since they arise exclusively from the Coulomb attraction of the electrons to the ions at their fixed positions. However, the Hamiltonian $\hat{H}(t)[n]$ does depend on time through the density, which we emphasize by the notation $\hat{H}[n(t)]$ in this work. In what follows, we refer by "self-consistency" to this aspect of the problem by which the instantaneous density must, in principle, be used at each step of the real-time integration.

In order to solve the TDKS equations (1), a set of initial conditions for the wave functions is necessary; in this work, they are represented by the perturbed ground-state wave functions. They are obtained by applying an initial perturbation (see below) to the solutions of the time-independent KS Hamiltonian.

\section{B. Energy conservation during the propagation}

The TDKS equations [cf. Eq. (1)] reduce to the KS equations in the case that the time dependence is factored out. In addition, within the adiabatic approximation they have the property to conserve the total energy $E(t)$,

$$
\begin{aligned}
E(t)= & \sum_{i}\left\langle\phi_{i}(t)|\hat{T}| \phi_{i}(t)\right\rangle \\
& +\int n(\mathbf{r}, t) V_{\mathrm{ext}}(\mathbf{r}, t) \mathrm{d} \mathbf{r}+E_{\mathrm{HXC}}[n](t),
\end{aligned}
$$

which is a generalization of the time-independent $\mathrm{KS}$ energy. ${ }^{20}$ For the case of HXC potentials that only depend on the instantaneous density, i.e., the adiabatic functional approximation, the conservation of the energy ${ }^{21}$ can be seen from the total time derivative of Eq. (3),

$$
\begin{aligned}
\frac{\mathrm{d} E(t)}{\mathrm{d} t}= & \sum_{i}\left\{\left\langle\phi_{i}(t)|\hat{T}| \frac{\mathrm{d}}{\mathrm{d} t} \phi_{i}(t)\right\rangle+\text { c.c. }\right\} \\
& +\sum_{i}\left\{\left\langle\phi_{i}(t)\left|\hat{V}_{\mathrm{ext}}(t)\right| \frac{\mathrm{d}}{\mathrm{d} t} \phi_{i}(t)\right\rangle+\text { c.c. }\right\} \\
& +\sum_{i}\left\{\left\langle\phi_{i}(t)\left|\left(\frac{\mathrm{d}}{\mathrm{d} t} \hat{V}_{\mathrm{ext}}(t)\right)\right| \phi_{i}(t)\right\rangle\right\} \\
& +\frac{\mathrm{d}}{\mathrm{d} t} E_{\mathrm{HXC}}[n(t)] .
\end{aligned}
$$

The last term requires some attention

$$
\begin{aligned}
\frac{\mathrm{d}}{\mathrm{d} t} E_{\mathrm{HXC}}[n(t)] & =\int \frac{\partial E_{\mathrm{HXC}}[n(t)]}{\partial n(\mathbf{r})} \frac{\partial n(\mathbf{r}, t)}{\partial t} \mathrm{~d} \mathbf{r} \\
& =\int V_{\mathrm{HXC}}^{\text {adiab }}[n(t)](\mathbf{r}) \frac{\partial n(\mathbf{r}, t)}{\partial t} \mathrm{~d} \mathbf{r} \\
& =\sum_{i}\left\{\left\langle\phi_{i}(t)\left|\hat{V}_{\mathrm{HXC}}^{\text {adiab }}[n(t)]\right| \frac{\mathrm{d}}{\mathrm{d} t} \phi_{i}(t)\right\rangle+\text { c.c. }\right\} .
\end{aligned}
$$


Inserting Eq. (5) into Eq. (4) and collecting terms to recover the KS Hamiltonian, we obtain

$$
\begin{aligned}
\frac{\mathrm{d}}{\mathrm{d} t} E(t)= & \sum_{i}\left\{\left\langle\phi_{i}(t)|\hat{H}[n(t)]| \frac{\mathrm{d}}{\mathrm{d} t} \phi_{i}(t)\right\rangle+\text { c.c. }\right\} \\
& +\int n(\mathbf{r}, t) \frac{\partial V_{\text {ext }}(\mathbf{r}, t)}{\partial t} \mathrm{~d} \mathbf{r} .
\end{aligned}
$$

Using the equations of motion (1), for a Hermitian KS Hamiltonian, it becomes clear that

$$
\begin{aligned}
& \left\langle\phi_{i}(t)|\hat{H}[n(t)]| \frac{\mathrm{d}}{\mathrm{d} t} \phi_{i}(t)\right\rangle+\text { c.c. } \\
& \quad=\mathrm{i} \hbar\left\langle\phi_{i}(t)|\hat{H}[n(t)] \hat{H}[n(t)]| \phi_{i}(t)\right\rangle+\text { c.c. } \\
& \quad=0
\end{aligned}
$$

and, hence, the sum of the first terms in Eq. (6) is zero. ${ }^{21}$ The last term in Eq. (6) also vanishes if the external potential does not depend on the time $t$, as it is the case for the systems considered here. Energy conservation is an important property of the TDKS equations since it can be used as a test of both stability and numerical accuracy of the numerical integration of the TDKS equations. This will be used as a test criterion in Secs. IV and V, in absence of exact non-trivial reference solutions to the TDKS problem in general.

\section{Plane-wave expansion of the wave functions}

For the numerical treatment of the TDKS equations, the KS orbitals need to be expanded in a finite number of basis functions. In this work, a supercell approach with periodic boundary conditions is used and, hence, the Bloch theorem ${ }^{22}$ can be exploited, leading to the expression

$$
\phi_{i}(\mathbf{r}, t)=\psi_{n \mathbf{k}}(\mathbf{r}, t)=\frac{1}{\sqrt{\Omega}} e^{i \mathbf{k} \cdot \mathbf{r}} u_{n \mathbf{k}}(\mathbf{r}, t)
$$

for the wave functions, where $u_{n \mathbf{k}}(\mathbf{r}, t)$ is a lattice-periodic function and $\Omega$ is the volume of the supercell. The indices $n$ and $\mathbf{k}$ label the eigenstates and the $\mathbf{k}$ vectors in the Brillouin zone, respectively. Expanding the wave functions into plane waves according to

$$
\psi_{n \mathbf{k}}(\mathbf{r}, t)=\frac{1}{\sqrt{\Omega}} \sum_{\mathbf{G}} C(\mathbf{G}, t) e^{\mathrm{i}(\mathbf{k}+\mathbf{G}) \cdot \mathbf{r}},
$$

where $\mathbf{G}$ belongs to the reciprocal lattice, has been quite successful in terms of numerical accuracy, convergence, and computational scalability. This basis set is orthonormal and provides an unbiased and spatially homogeneous expansion that converges numerically by systematically increasing merely a single parameter, the cutoff energy $E_{\text {cut }}=G_{\text {cut }}^{2} / 2$. $E_{\text {cut }}$ corresponds to the maximum kinetic energy of all plane waves in the calculation.

Since the basis itself is time independent, all the time dependence is carried by the plane-wave coefficients $C(\mathbf{G}, t)$ of the expansion. This implies that the spatial partial derivatives of the wave functions are readily available in the reciprocal space. It is also useful to note that the plane-wave basis is independent of the nuclei positions, which makes the frame- work convenient for first-principles molecular dynamics approaches, thanks to the absence of basis set superposition errors and Pulay forces. ${ }^{23}$

\section{Computational details}

Treating the singularity of the Coulomb potential in the close vicinity of the nuclei is computationally expensive in a plane-wave basis since a very large number of basis functions is needed for converged calculations. The pseudopotential approach is used to circumvent this issue by replacing the core electrons with non-local effective potentials, which are derived by inverting the atomic KS equation (see, e.g., Ref. 24). In the present work, Hamann-SchlüterChiang norm-conserving pseudopotentials with a modification by Vanderbilt ${ }^{25}$ are used within the Kleinman-Bylander approach. ${ }^{26}$

The local-density approximation as parametrized by Perdew and Zunger ${ }^{27}$ is used within the adiabatic approximation for the exchange-correlation potential in the calculations. Due to the size of the supercells in this work, it is sufficient to use only the $\Gamma$ point to sample the Brillouin zone even though the implementation is capable of taking $\mathbf{k}$ points into account.

\section{PROPAGATION SCHEMES}

\section{A. General considerations}

For a non-interacting time-dependent Schrödinger equation (TDSE) there exists a plethora of numerical propagators to choose from. Their key properties such as (semi-exact) norm conservation and (semi-exact) energy conservation as well as other numerical properties including different conditions of stability (with respect to the time/space discretization) are well known. ${ }^{28}$ In spite of the similarities, for the case of the TDKS equations (1), in combination with a plane-wave expansion of the wave functions, the situation is complicated by several factors:

First, unlike the TDSE, the TDKS equations are nonlinear, and the inverse of the KS operator is only available through numerical iterative solution.

Second, conditional or unconditional stability of a time propagation algorithm in the case of the TDSE is not a guarantee of stability for the TDKS case; due to the self-consistent nature of the TDKS, there are feedback mechanisms that can worsen the stability criterion, or even make a method completely unstable when applied to the TDKS. This is a well-known phenomenon observed in studies of the nonlinear extensions of the Schrödinger equation ${ }^{29,30}$ and will be discussed for the second-order finite-differences scheme below.

Third, within the plane-wave framework, implicit methods are not suitable due to the dimension of the linear operator (number of plane waves squared) that has to be inverted. The number of plane waves in the present calculations is over 33000 , and is routinely much larger for many applications. Therefore, for example, the Crank-Nicholson (Cayley) scheme, ${ }^{31}$ although it has been successfully used in reduced basis set local-orbital methods (see, e.g., Refs. 12 and 32), is not a desirable option for large-scale simulations (thousands of electrons) within a plane-wave implementation because a 
linear solver has to be used for inverting the large matrix at every step. ${ }^{33}$ It is certainly possible to have such implicit methods implemented for a plane-wave basis, however, the accuracy goal of the iterative solver becomes an extra parameter in itself, to be controlled to ensure stability. Therefore, implicit methods are beyond the scope of this work.

Fourth, within the plane-wave formalism it is tempting to use approximations to the linear time-evolution operator instead of approximating the differential equation by finite differences. Moreover, split-operator Trotter-like expansions exploit the fact that the kinetic energy (the local potential) part of the Hamiltonian is diagonal in reciprocal (real) space. Since these techniques can potentially benefit from very large time steps, ${ }^{10}$ they have been traditionally favored in the community. ${ }^{9}$ However, these approaches cannot be implemented straightforwardly without further algorithmic considerations because one needs to deal with the non-local part of the pseudopotential. Furthermore, the Hamiltonian operator is not a constant and not known a priori, since it depends on the electronic states at later times (see, e.g., the Magnus expansion ${ }^{34}$ and the railway method ${ }^{10}$ ). Hence, in this work we restrict ourselves to finite-difference approximations which have a controlled order in the error. Nevertheless, the time steps that are used in this work, are comparable to the ones used in other approaches. ${ }^{14,35-39}$

Note also that all propagators investigated in this work rely on the application of the entire Hamiltonian to the wave function and on updating the Hamiltonian correspondingly; therefore, no particular structure of the Hamiltonian is assumed or exploited for the propagation. Consequently, for the integration of the TDKS equations we can directly take advantage of some of the existing DFT architecture as implemented in the Qbox code (or similar plane-wave codes). This code is optimized for scalability and features highly optimized routines for solving the regular KS equations on a large number of processors. ${ }^{40}$

\section{B. First-order Euler scheme}

The Euler approach is the simplest propagation scheme in which the wave functions at the time $t+\Delta t$ are obtained from the one at time $t$ according to

$$
|\phi(t+\Delta t)\rangle=|\phi(t)\rangle-\frac{\mathrm{i}}{\hbar} \Delta t \hat{H}[n]|\phi(t)\rangle .
$$

For this scheme, only the static Hamiltonian at $t=0$ (i.e., $\left.\hat{H}\left[n(t=0)=n_{0}\right]\right)$ is used to drive the time propagation in Eq. (10). While for any real, i.e., physical problem, the selfconsistent Hamiltonian that depends on time through the density $n(t)$ has to be used, we explore numerical properties of the propagation given by Eq. (10) using only the fixed Hamiltonian in this work. This is due to the significant numerical instability issues that we observed for this scheme and discuss below. The computational load associated with solving Eq. (10) arises from a single application of the Hamiltonian to the wave functions. The error in this scheme is $\mathscr{O}(\Delta t)$, i.e., first-order in $\Delta t$.

\section{Second-order finite differences}

Within the second-order finite-difference scheme (hereafter called the SOD scheme) more information than in the Euler scheme is used to calculate the wave functions at $t+\Delta t$ from the ones at both $t$ and $t-\Delta t$ according to

$$
|\phi(t+\Delta t)\rangle=|\phi(t-\Delta t)\rangle-2 \frac{\mathrm{i}}{\hbar} \Delta t \hat{H}[n]|\phi(t)\rangle .
$$

SOD is slightly more sophisticated than the Euler approach, and the error is $\mathscr{O}\left(\Delta t^{2}\right)$. As in the case of the Euler scheme, the Hamiltonian has to be applied only once for each time step, but two copies of the wave functions (at $t$ and $t-\Delta t$ ) need to be stored in memory for the calculation of the new wave functions. The SOD case presents an interesting comparison since this scheme is known to be conditionally stable with semi-exact conservation of energy and orthogonality for the non-self-consistent (TDSE) problem.

For the propagation according to Eq. (11), three different levels of the self-consistency of the Hamiltonian ${ }^{28}$ are compared, again, in order to study the different numerical properties for three cases. In the fully self-consistent approach (sc-SOD), the Hamiltonian is updated at each time step (cf. Eq. (11)), as it is required when a physical problem is to be studied. However, in practical applications where the electronic wave functions are spread out in the cell, the density (as an average of electronic wave functions squared) often washes out the individual high-frequency fluctuations of the wave functions, giving in practice a quantity that has a lower frequency. Hence, we consider two additional cases: (i) Within the non-self-consistent propagation (nsc-SOD) the Hamiltonian is kept fixed at $\hat{H}\left[n_{0}\right]$, as described for the Euler scheme above. (ii) In the semi-self-consistent propagation (sc100SOD) the Hamiltonian is updated every 100 time steps, i.e., self-consistency is recovered every 100 steps. By assessing the numerical stability and accuracy associated with the nonlinearity for the sc100-SOD we elucidate whether this scheme can be a useful tool for cases where the density fluctuations are of a lower frequency compared to individual electron dynamics.

\section{Second-order Runge-Kutta scheme}

Another second-order approach tested in this work is the second-order Runge-Kutta ${ }^{31}$ scheme (called RK2 in the following). In this case, the wave functions at $t+\Delta t$ are computed according to

$$
\begin{aligned}
& \left|k_{1}\right\rangle=-\frac{\mathrm{i}}{\hbar} \Delta t \hat{H}\left[n_{\phi(t)}\right]|\phi(t)\rangle, \\
& \left|k_{2}\right\rangle=-\frac{\mathrm{i}}{\hbar} \Delta t \hat{H}\left[n_{\phi(t)+0.5 \cdot k_{1}}\right]\left|\phi(t)+0.5 \cdot k_{1}\right\rangle, \\
& |\phi(t+\Delta t)\rangle=\left|\phi(t)+k_{2}\right\rangle .
\end{aligned}
$$

As with the SOD scheme, the integration error scales as $\mathscr{O}\left(\Delta t^{2}\right)$. The higher sophistication of this approach with respect to the Euler scheme is achieved by invoking multiple evaluations of the Hamiltonian and deriving the updated wave functions from these intermediate steps. ${ }^{31}$ In or- 
der to express how the density needs to be computed for the different intermediate steps, we use the notation $n_{\phi(t)}$ in Eqs. (12) and (13). While the RK2 method allows for larger time steps for the integration of the TDKS equations, it also comes with higher computational cost due to the additional updates of the Hamiltonian and its application to the respective wave functions. Furthermore, we emphasize that, unlike in Secs. III B and III C, the Hamiltonian is updated at each time step for the time propagation schemes described in Secs. III D and III E.

\section{E. Fourth-order Runge-Kutta scheme}

The fourth-order Runge-Kutta scheme (RK4) is a step beyond the RK2 method, further improved by including more intermediate evaluations of the Hamiltonian. ${ }^{31}$ In this case, the propagation is done according to

$$
\begin{aligned}
& \left|k_{1}\right\rangle=-\frac{\mathrm{i}}{\hbar} \Delta t \hat{H}\left[n_{\phi(t)}\right]|\phi(t)\rangle, \\
& \left|k_{2}\right\rangle=-\frac{\mathrm{i}}{\hbar} \Delta t \hat{H}\left[n_{\left.\phi(t)+0.5 \cdot k_{1}\right]\left|\phi(t)+0.5 \cdot k_{1}\right\rangle,}\right. \\
& \left|k_{3}\right\rangle=-\frac{\mathrm{i}}{\hbar} \Delta t \hat{H}\left[n_{\left.\phi(t)+0.5 \cdot k_{2}\right]\left|\phi(t)+0.5 \cdot k_{2}\right\rangle,}\right. \\
& \left|k_{4}\right\rangle=-\frac{\mathrm{i}}{\hbar} \Delta t \hat{H}\left[n_{\phi(t)+k_{3}}\right]\left|\phi(t)+k_{3}\right\rangle, \\
& |\phi(t+\Delta t)\rangle=\left|\phi(t)+\frac{1}{6} k_{1}+\frac{1}{3} k_{2}+\frac{1}{3} k_{3}+\frac{1}{6} k_{4}\right\rangle .
\end{aligned}
$$

The RK4 scheme clearly shows the highest computational cost, as it requires four evaluations and updates of the Hamiltonian. On the other hand, for a given local error tolerance, the time step can be chosen much larger because the integration error only scales as $\mathscr{O}\left(\Delta t^{4}\right)$. In practice, other restrictive considerations, like stability, have to be taken into account to choose the time step for each method, as discussed below.

\section{TEST CASE I: THE ISOLATED NA ATOM}

As a test of the propagators introduced in Sec. III, the time-evolution of a single $\mathrm{Na}$ atom in a cubic supercell ( $a=7.94 \AA$ ) is studied (cf. Fig. 1). The size of this supercell, i.e., the distance between mirror images of the $\mathrm{Na}$ atom, has been chosen such that the total energy does not change more than $55 \mathrm{meV}$ upon a further increase of the cell size.

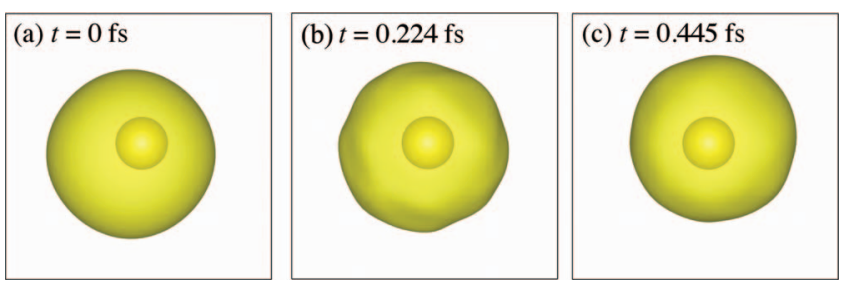

FIG. 1. Cubic unit cell containing a single Na atom (small circle). To prepare a non-equilibrium initial condition, the $\mathrm{Na} 3 s$ wave function (represented by the yellow isosurface) has been shifted by $(0.32,0.32,0.32) \AA$, see (a). In (b) and (c), two snapshots of the RK4 simulation are shown.

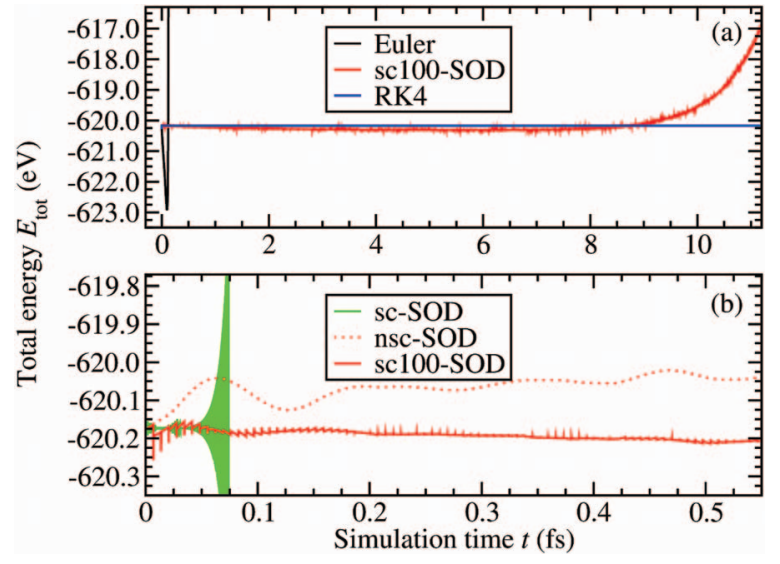

FIG. 2. Total energy $E_{\mathrm{tot}}$ (in $\mathrm{eV}$ ) of the $\mathrm{Na}$ atom (at $t=0 \mathrm{fs}$, the $3 \mathrm{~s}$ wave function was shifted by $(0.32,0.32,0.32) \AA$ from its equilibrium position in real space) as a function of time $t$ (in fs). In (a), the Euler scheme (black solid line, $\Delta t=0.069$ as) and the sc100-SOD second-order finite-difference scheme (red solid line, $\Delta t=0.069$ as) are compared to the Runge-Kutta propagators (blue solid line). The second-order ( $\Delta t=0.069$ as) and the fourth-order ( $\Delta t=0.691$ as) Runge-Kutta scheme yield the same trajectory for the times shown in (a). In (b), the fully self-consistent second-order finite-difference method (green solid line) is compared to the sc100-SOD (red solid line) and the non-self-consistent (red dotted line) one for $\Delta t=0.069$ as.

The plane-wave cutoff energy was chosen to be 70 Ry which ensures that the total energy is converged to about $20 \mathrm{meV}$. For this system, the ground-state density is calculated within DFT and, subsequently, the $3 s$ wave function is shifted by $(0.32,0.32,0.32) \AA$ in real space in order to prepare a nonequilibrium initial condition for the time propagation.

\section{A. Numerical stability and conservation of energy}

The numerical stability is one of the most important criteria when solving the TDKS equations with the explicit integrators introduced above. Since the total energy is a conserved quantity, as discussed in Sec. II B, it is used as a measure of the stability of the propagation.

In Fig. 2, the time evolution of the total energy is shown for the different schemes introduced in Sec. III. A time step of $\Delta t=0.069$ as is used for all schemes except for the RK4 one; in this case a ten times larger time step, $\Delta t=0.69$ as, was used. We note that this is on the same order of magnitude as the time steps of one ${ }^{11}$ or a few ${ }^{35}$ attoseconds reported for real-space and real-time codes.

From Fig. 2(a) it can be seen that the Euler scheme, even though the Hamiltonian is kept fixed during the propagation, is highly unstable. The total energy diverges very quickly after a decrease from its original value. In the same figure, the results obtained using the RK2 propagator are in agreement with the RK4 propagator and it is found that they conserve the total energy within $2.72 \mathrm{meV}$ for a propagation time of up to $11 \mathrm{fs}$. Even though both of the two propagation schemes appear to fulfill the numerical stability, the RK2 scheme eventually becomes unstable for a longer propagation as discussed below. For the RK4 approach, also the conservation of the norm of the unshifted wave functions was checked; we found the (monotonous) decrease of the norm to be smaller than $10^{-7} /$ fs during the propagation. In addition, Fig. 2(a) shows 
that the sc100-SOD scheme is able to maintain the stability to a certain point: Up until $\approx 8.5$ fs the total energy is conserved fairly well and is only slightly smaller than the initial value. However, beyond that point the deviation grows very quickly and the total energy again diverges.

For comparison, the different levels of self-consistency in the SOD scheme as shown in Fig. 2(b) are analyzed. The figure clearly shows that the sc-SOD scheme is numerically unstable even for a propagation time of less than $0.1 \mathrm{fs}$. Keeping the Hamiltonian fixed for some number of integration steps (e.g., 100 in this work) improves the numerical stability of the algorithm, leading to a stable propagation for a much longer time [cf. Fig. 2(a)]. The nsc-SOD scheme is found to be conditionally stable, i.e., there is a $\Delta t$ small enough such that the propagation remains stable for all times $t$. However, keeping the Hamiltonian fixed is unphysical for practical applications within RT-TDDFT, as mentioned earlier. Therefore, the SOD schemes are an example of an explicit method that is wellbehaved in the non-self-consistent case (or, for instance, the linear Schrödinger equation) but becomes unconditionally unstable for the integration of the self-consistent TDKS. This illustrates the difficulty of having to deal with the nonlinear Hamiltonian of the TDKS formalism when the time propagation is performed.

For investigating further details, Fig. 3 shows the numerical stability of the RK2 (the sc100-SOD second-order) propagation schemes up to $35 \mathrm{fs}$ ( $11 \mathrm{fs}$ ) as calculated using different time steps $\Delta t$. For both propagators, the propagation becomes expeditiously more stable when smaller time steps are used. In addition, from comparing Figs. 3(a) and 3(b) it is found that the (fully self-consistent) RK2 scheme is much more stable than the sc100-SOD when the same time step is chosen. The total energy deviations in the stable regime are also much smaller for the RK2 case.

As mentioned earlier, the nsc-SOD and the RK4 propagator are found to be conditionally stable in this work, i.e., they can be used for propagation of arbitrary duration as long as the time step $\Delta t$ is chosen to be small enough. Plotting the critical time step (for which the propagation is numerically

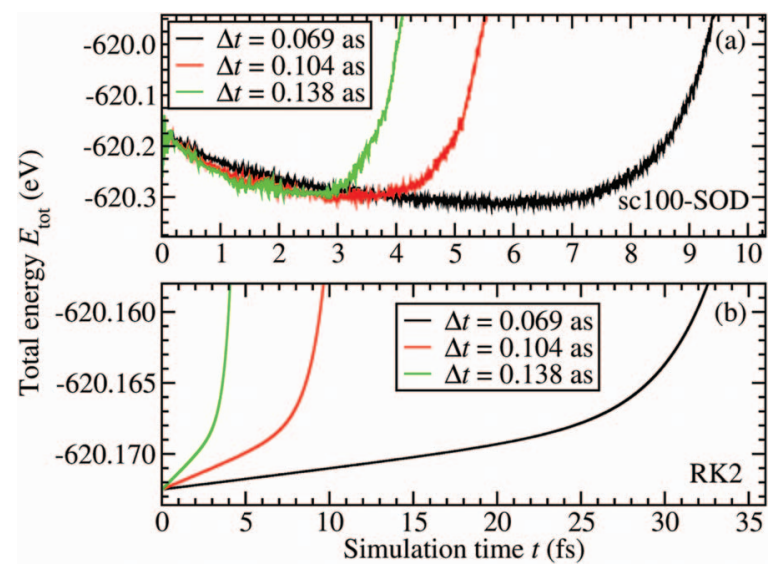

FIG. 3. Total energy $E_{\mathrm{tot}}$ (in $\mathrm{eV}$ ) of the $\mathrm{Na}$ atom as a function of time $t$ (in fs). The curves in (a) result from the sc100-SOD second-order finite-difference scheme and the ones in (b) from the second-order Runge-Kutta scheme. Time steps of $\Delta t=0.069$ as (black solid lines), $\Delta t=0.104$ as (red solid lines), and $\Delta t=0.138$ as (green solid lines) were used.

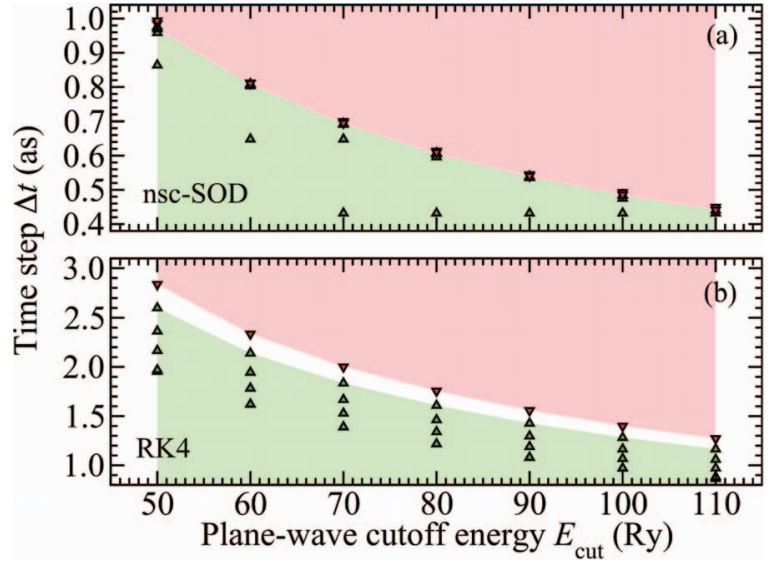

FIG. 4. Time steps $\Delta t$ (in as) for which the non-self-consistent second-order finite-difference scheme (a) or the fourth-order Runge-Kutta scheme (b) are stable (green triangles pointing up) or unstable (red triangles pointing down), depending on the respective plane-wave cutoff energy $E_{\text {cut }}$ (in Ry) used.

found to remain stable) versus the plane-wave cutoff energy $E_{\text {cut }}$ in Fig. 4 indicates an inverse proportionality of the critical $\Delta t$ vs. $E_{\text {cut }}$. Furthermore, it can be seen that the critical time step used in the RK4 scheme can be roughly three times as large as the one used in the nsc-SOD for a given $E_{\text {cut }}$. We emphasize, however, that the nsc-SOD scheme is not physically meaningful for propagating the TDKS equation even though it is numerically robust.

\section{B. Evolution of the system}

In order to illustrate the evolution of the system during the propagation, the total energy, being a conserved quantity, does not provide helpful insights. Instead, the sum of the expectation values, $\sum_{i}\left\langle\phi_{i}(t)|\hat{H}[n]| \phi_{i}(t)\right\rangle$, as shown in Fig. 5

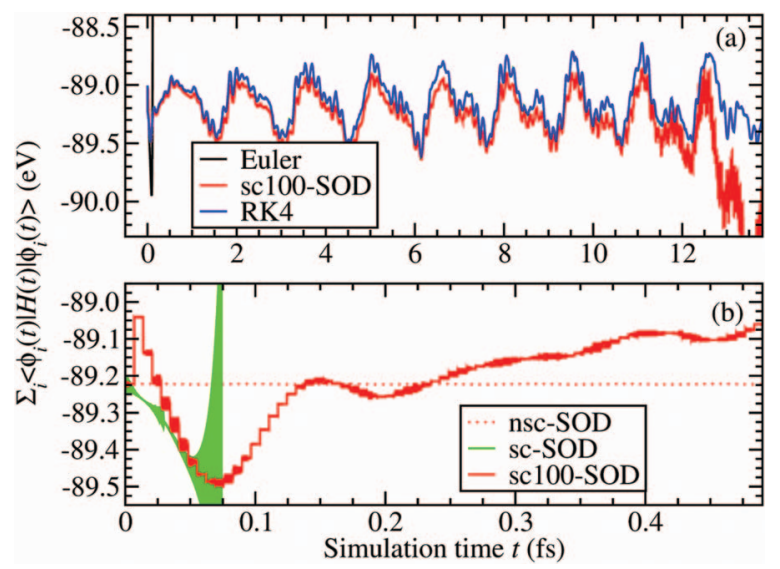

FIG. 5. Sum of the expectation values $\sum_{i}\left\langle\phi_{i}(t)|\hat{H}[n]| \phi_{i}(t)\right\rangle$ (in eV) of all valence wave functions $i$ of the $\mathrm{Na}$ atom (at $t=0$ fs the $3 \mathrm{~s}$ wave function was shifted by $(0.32,0.32,0.32) \AA$ from its equilibrium position in real space) as a function of time $t$ (in fs). In (a) the Euler scheme (black solid line, $\Delta t=0.069$ as) and the sc100-SOD second-order finite-difference scheme (red solid line, $\Delta t=0.069$ as) are compared to the Runge-Kutta propagators (blue solid line). The second-order ( $\Delta t=0.069$ as) and the fourth-order ( $\Delta t=0.691$ as) Runge-Kutta scheme yield the same trajectory for the times shown in (a). In (b) the fully self-consistent second-order finite-difference method (green solid line) is compared to the sc100-SOD (red solid line) and the non-selfconsistent (red dotted line) one for $\Delta t=0.069$ as. 
is used as a time-dependent quantity that provides qualitative information of the energy spectrum of the wave functions. As expected, the results from the Euler scheme are not useful due to the immediate instability of this propagation scheme. For $t$ up to $\approx 13$ fs, it can be seen in Fig. 5(a) that the RK2 and RK4 propagations agree with each other exactly (therefore only RK4 is shown) even though they are calculated using different $\Delta t$. They show pronounced oscillations with a period of approximately 1.5 fs. Additionally, contributions from other higher frequencies become visible from the fine structure of the curves. These features derive from the fact that the wave function that was shifted at $t=0$ starts to oscillate around the (fixed) position of the nucleus, as expected. Also other wave functions start to oscillate through the self-consistent potential (which depends on all occupied wave functions) in the TDKS Hamiltonian.

We note that test calculations have shown that varying the plane-wave cutoff energy between 50 Ry and 80 Ry does not significantly impact the time evolution of the system with the change in the sum of the expectation values remaining smaller than $8 \mathrm{meV}$.

Comparing the results of the two Runge-Kutta propagators [cf. Fig. 5(a)] to the behavior obtained using the sc100SOD propagation scheme shows a close similarity for small times $t$. However, this agreement becomes worse for larger times, i.e., when the sc100-SOD becomes increasingly unstable. In accordance with the discussion of the total energy, Fig. 5(b) points out that the sc-SOD becomes unstable and, hence, does not yield meaningful results for the expectationvalue sum for $t>0.1 \mathrm{fs}$. Even though the nsc-SOD is conditionally stable, Fig. 5(b) shows that the expectation-value sum does not change during the propagation because of the non-self-consistent nature of the propagation.

Figure 6 shows the long-term stability of the propagation using the RK4 scheme; the total energy for the $\mathrm{Na}$ atom is plotted for a total propagation time of $2.2 \mathrm{ps}$. It can be seen that the total energy is well conserved during the propagation with a deviation of less than $30 \mathrm{meV}$ during the $2.2 \mathrm{ps}$. As discussed above, the integration error in the RK4 scheme scales as $\mathscr{O}\left(\Delta t^{4}\right)$. More specifically, it has been found numerically that the total energy conservation (per unit time) can be improved by a factor of 27.8 when the time step is reduced by a half.

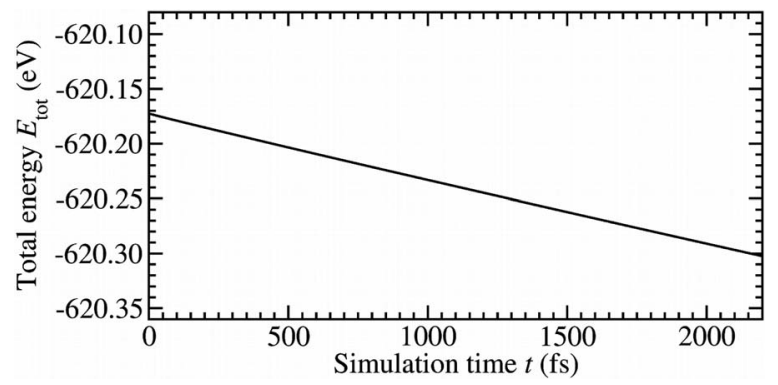

FIG. 6. Total energy $E_{\mathrm{tot}}$ (in $\mathrm{eV}$ ) of the $\mathrm{Na}$ atom (at $t=0$ fs the $3 s$ wave function was shifted by $(0.32,0.32,0.32) \AA$ from its equilibrium position in real space) as a function of time $t$ (in fs). The results have been obtained using the RK4 propagator and $\Delta t=0.691$ as.

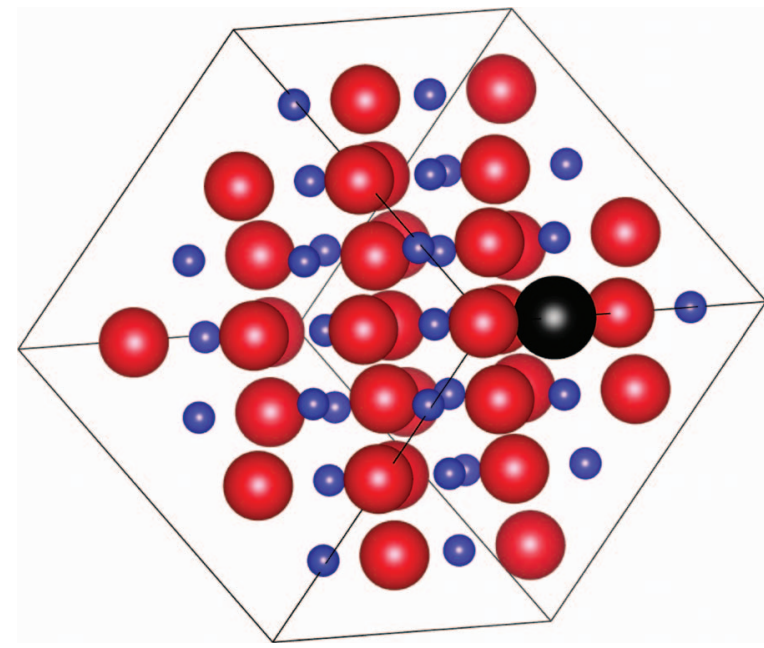

FIG. 7. The 64-atom unit cell of $\mathrm{MgO}$ ( $\mathrm{Mg}$ atoms red circles, $\mathrm{O}$ atoms blue circles) containing a single $\mathrm{Na}$ atom (black circle) on an oxygen lattice position.

\section{TEST CASE II: NA ATOM EMBEDDED IN MGO}

Having established that the RK4 method is suitable for our implementation of RT-TDDFT within a plane-wave pseudopotential formalism, we now show that the scheme is suitable for investigating large, complex systems and, in particular, bulk systems with periodic boundary conditions. This aspect is a major improvement over most existing implementations of RT-TDDFT. We applied our implementation to a bulk system, consisting of a 64-atom supercell of crystalline $\mathrm{MgO}$ with one of the $\mathrm{O}$ atoms replaced by a $\mathrm{Na}$ atom. This corresponds to $32 \mathrm{Mg}$ atoms, $31 \mathrm{O}$ atoms, and $1 \mathrm{Na}$ atom with a total of 449 valence electrons in the calculation (cf. Fig. 7). After obtaining the ground state of the system, the $3 s$ wave function of the $\mathrm{Na}$ atom is shifted by $(0.032,0.032,0.032) \AA$ in real space to obtain a non-equilibrium initial condition for the time propagation.

The evolution of the $\mathrm{Na}: \mathrm{MgO}$ system is shown in Fig. 8 using again the sum of the expectation values as an example for a quantity of interest. The sc100-SOD and the RK2 are

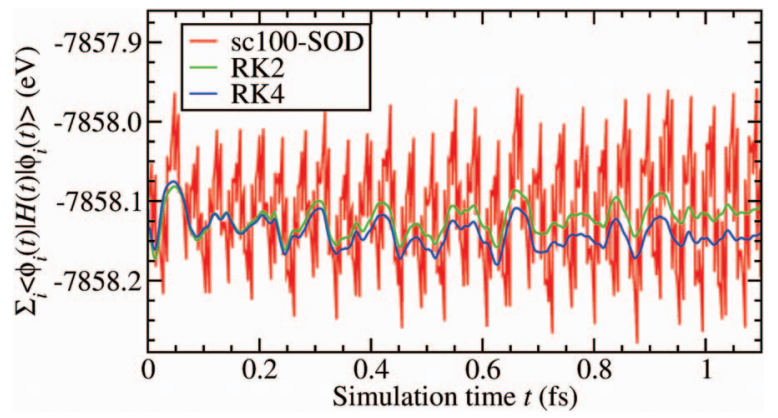

FIG. 8. Sum of the expectation values $\sum_{i}\left\langle\phi_{i}(t)|\hat{H}[n]| \phi_{i}(t)\right\rangle$ (in eV) of all valence wave functions in the $\mathrm{Na}: \mathrm{MgO}$ 64-atom supercell (at $t=0$ fs the Na-induced level within the $\mathrm{MgO}$ gap was shifted by $(0.032,0.032,0.032)$ $\AA$ from its equilibrium position in real space) as a function of time $t$ (in fs). The sc100-SOD second-order finite-difference scheme (red solid line, $\Delta t=0.069$ as) is compared to the second-order (green solid line, $\Delta t$ $=0.069$ as) and the fourth-order (blue solid line, $\Delta t=0.691$ as) Runge-Kutta scheme. 


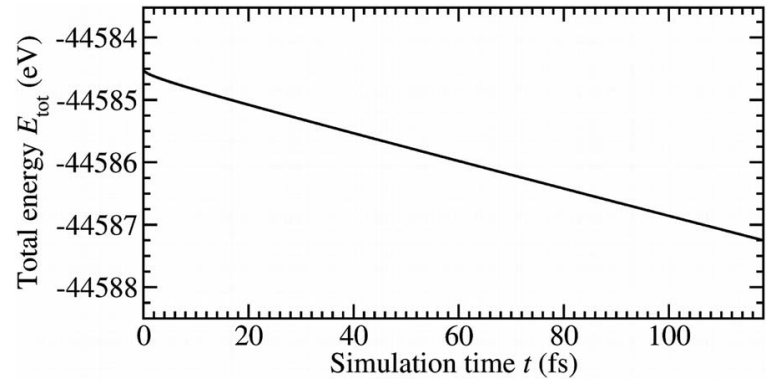

FIG. 9. Total energy $E_{\text {tot }}$ (in eV) of the Na:MgO 64-atom supercell (at $t=0$ fs the Na-induced level within the $\mathrm{MgO}$ gap was shifted by $(0.032$, $0.032,0.032) \AA$ from its equilibrium position in real space) as a function of time $t$ (in fs). The results have been obtained using the fourth-order RungeKutta propagator and $\Delta t=0.691$ as

also shown for comparison to the RK4 propagation scheme. The intrinsic inaccuracy of the sc100-SOD scheme is evident in the figure as this propagator is applied to a complex system such as $\mathrm{Na}: \mathrm{MgO}$ : the oscillations are much too large and the long time stability is worse, as discussed earlier.

To test the long-term stability of the RK4 propagation also for this complex system, we plotted the total energy for a total propagation time of $118 \mathrm{fs}(\Delta t=0.691$ as) in Fig. 9. The total energy is well-conserved during the propagation; the deviation remains on the order of $2.7 \mathrm{eV}$ during $118 \mathrm{fs}$. Note that this error corresponds to merely $0.006 \%$ of the total energy of the system, hence, the magnitude of the deviation per electron is on the same order as in the case of a single $\mathrm{Na}$ atom. Furthermore, as discussed above it can be reduced by a factor of $\approx 28$ by using a half of the time step size.

\section{A. Parallel scaling}

Finally, it is important to analyze the scaling of the RTTDDFT implementation presented in this work. In Fig. 10, the time required to perform one step of real time propagation using the RK4 method is shown for the different numbers of processor cores the calculation is run on. As discussed above, the RK4 method requires four updates of the Hamiltonian and four evaluations of $\hat{H}\left[n_{\phi(t)}\right]|\phi(t)\rangle$ per time step. In Fig. 10, we also compare the time required to perform one step of steepest descent (SD), which requires one evaluation of the Hamilto-

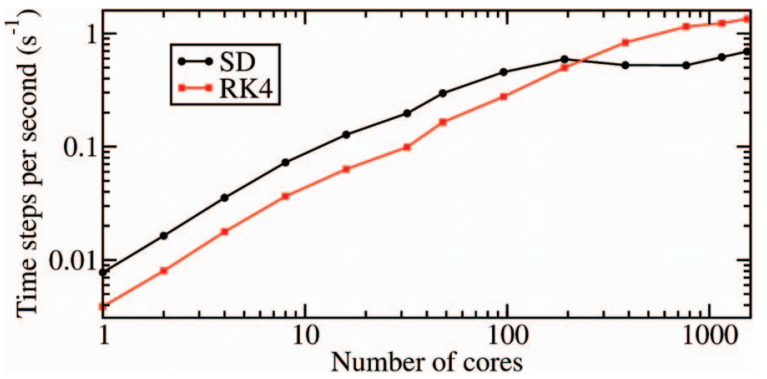

FIG. 10. The number of steps that can be performed within $1 \mathrm{~s}$ wall time is plotted versus the number of processing cores used in the calculation for the $\mathrm{Na}: \mathrm{MgO}$ 64-atom supercell. The steepest descent algorithm (black curve) is compared to the RK4 propagation (red curve). nian per step but also one orthogonalization of the wave functions. Even though there are more sophisticated schemes than SD that can be used for converging to the ground-state Hamiltonian (e.g., conjugate-gradient, preconditioning, etc.), these schemes all involve orthogonalization of the wave functions. We chose the SD algorithm merely as a representative case of these schemes, since the orthogonalization of the wave functions does not scale well with the number of processing cores and, hence, represents a severe bottleneck.

Initially, for small numbers of processor cores, the SD scheme is roughly a factor of four faster since only one evaluation of the Hamiltonian is required. However, the orthogonalization bottleneck of the SD scheme becomes obvious in Fig. 10 for more than 200 cores, where the scalability of the SD scheme is significantly reduced. In contrast, the RK4 propagator scales very well (close to linearly) up to at least 1536 cores (1 step/s), benefiting directly from the highly parallelized routines in the Qbox code that can be exploited for this explicit integration scheme. The absence of an orthogonalization bottleneck in the RK4 scheme leads to highly parallel calculations in addition to the fact that having an explicit integrator scheme allows us to take advantage of the highly parallel routines for applying Hamiltonian on the wave functions. Indeed, several authors have noted that under suitable conditions a coupled electron-nuclear dynamics within TDDFT could outperform Born-Oppenheimer molecular dynamics. ${ }^{14,41}$ In this work, we show an instance of these conditions, namely, large numbers of electrons and a highly parallel implementation.

\section{CONCLUSIONS}

We find that the 4th order Runge-Kutta scheme is a conditionally stable and well-balanced general purpose propagator for the TDKS equations when implemented within the planewave pseudopotential formalism. Several other integrators such as the Euler scheme, the second-order finite-differences scheme, and the second-order Runge-Kutta scheme were also studied in this work. The Euler scheme was found to be highly unstable. Relaxing the self-consistency requirement of the nonlinear Hamiltonian in the TDKS improves the stability of the second-order finite differences scheme. This observation indicates that integrators that are designed for time-dependent Schrödinger equations are not necessarily suitable for integrating TDKS equations. In limited instances, the second-order Runge-Kutta scheme can be an efficient alternative propagator (with smaller computational expense than the fourth-order version) for cases where the time step needs to be very small and the total propagation time required is not long. This can be the case for certain applications involving very fast external perturbations where the physics of the problem requires a very fine time resolution (e.g., under a high frequency external potential).

The explicit propagators allow for a better scalability with respect to the number of computing cores compared to typical ground-state or Born-Oppenheimer methods, making them suitable for large-scale simulations. We show in this work the precise regime in which a single real time step propagation can outperform a BO method step. The improvement 
results from the fact that the time-propagation schemes do not require the orthogonalization of the propagated wave functions, which is a known bottleneck for the scalability. ${ }^{17}$ Furthermore, the explicit integrators do not assume a particular form of the potential (including the pseudopotential and exchange-correlation potential) and they can directly benefit from existing highly parallel routines developed for the BornOppenheimer calculations.

The present implementation of real-time TDDFT in the plane-wave pseudopotential formalism using the explicit integrators provides an ideal framework for performing largescale first-principles Ehrenfest dynamics simulations, which will be discussed in a separate future work with extensive applications.

\section{ACKNOWLEDGMENTS}

We gratefully acknowledge fruitful discussions with E. K. U. Gross, A. Rubio, K. Burke, N. Maitra, and J. Kohanoff. A.S. is supported through the Physical and Life Sciences Directorate at Lawrence Livermore National Laboratory. Computing support for this work came from the Lawrence Livermore National Laboratory (LLNL) Institutional Computing Grand Challenge program. Part of this work was performed under the auspices of the U.S. Department of Energy at Lawrence Livermore National Laboratory under Contract No. DE-AC52-07A27344.

${ }^{1}$ Y. Kanai, Z. Wu, and J. C. Grossman, J. Mater. Chem. 20, 1053 (2010).

${ }^{2}$ A. A. Correa, J. Kohanoff, E. Artacho, D. Sánchez-Portal, and A. Caro, Phys. Rev. Lett. 108, 213201 (2012).

${ }^{3}$ A. Caro and M. Victoria, Phys. Rev. A 40, 2287 (1989).

${ }^{4}$ M. Born and J. R. Oppenheimer, Ann. Phys. 389, 457 (1927).

${ }^{5}$ P. Ehrenfest, Z. Phys. A: Hadrons Nucl. 45, 455 (1927).

${ }^{6} \mathrm{~J}$. C. Tully, in Modern Methods for Multidimensional Dynamics Computations in Chemistry, edited by D. L. Thompson (World Scientific, 1998).

${ }^{7}$ E. Runge and E. K. U. Gross, Phys. Rev. Lett. 52, 997 (1984).

${ }^{8}$ P. Hohenberg and W. Kohn, Phys. Rev. 136, B864 (1964).

${ }^{9}$ M. Marques and E. Gross, Annu. Rev. Phys. Chem. 55, 427 (2004).

${ }^{10}$ O. Sugino and Y. Miyamoto, Phys. Rev. B 59, 2579 (1999).

${ }^{11}$ M. A. Marques, A. Castro, G. F. Bertsch, and A. Rubio, Comput. Phys. Commun. 151, 60 (2003).

${ }^{12}$ A. Castro, M. A. L. Marques, and A. Rubio, J. Chem. Phys. 121, 3425 (2004).
${ }^{13}$ A. Castro, and M. Marques, in Time-Dependent Density Functional Theory, Lecture Notes in Physics Vol. 706, edited by M. Marques, C. Ullrich, F. Nogueira, A. Rubio, K. Burke, and E. Gross (Springer, Berlin, 2006), pp. 197-210.

${ }^{14}$ X. Andrade, A. Castro, D. Zueco, J. L. Alonso, P. Echenique, F. Falceto, and Á. Rubio, J. Chem. Theory Comput. 5, 728 (2009).

${ }^{15}$ M. Casida, in Recent Advances in Density Functional Methods, Part I, edited by D. P. Chong (World Scientific, Singapore, 1995), p. 155.

${ }^{16}$ W. Kohn and L. J. Sham, Phys. Rev. 140, A1133 (1965).

${ }^{17}$ F. Gygi, IBM J. Res. Dev. 52, 137 (2008).

${ }^{18}$ F. Gygi, R. Yates, J. Lorenz, E. Draeger, F. Franchetti, C. Ueberhuber, B. de Supinski, S. Kral, J. Gunnels, and J. Sexton, in Proceedings of the ACM/IEEE SC 2005 Conference on Supercomputing (IEEE Computer Society, 2005), p. 24

${ }^{19}$ E. K. U. Gross, F. J. Dobson, and M. Petersilka, Density Functional Theory (Springer, New York, 1996).

${ }^{20}$ R. M. Martin, Electronic Structure: Basic Theory and Practical Methods, 1st ed. (Cambridge University Press, 2008), Vol. 1.

${ }^{21}$ E. K. U. Gross, private communication (2011).

${ }^{22}$ F. Bloch, Z. Phys. A 52, 555 (1929).

${ }^{23}$ P. Pulay, Mol. Phys. 17, 197 (1969).

${ }^{24}$ M. Fuchs and M. Scheffler, Comput. Phys. Commun. 119, 67 (1999).

${ }^{25}$ D. Vanderbilt, Phys. Rev. B 32, 8412 (1985).

${ }^{26}$ L. Kleinman and D. M. Bylander, Phys. Rev. Lett. 48, 1425 (1982).

${ }^{27}$ J. P. Perdew and A. Zunger, Phys. Rev. B 23, 5048 (1981).

${ }^{28}$ C. Leforestier, R. H. Bisseling, C. Cerjan, M. D. Feit, R. Friesner, A. Guldberg, A. Hammerich, G. Jolicard, W. Karrlein, H. D. Meyer, N. Lipkin, O. Roncero, and R. Kosloff, J. Comput. Phys. 94, 59 (1991).

${ }^{29}$ W. Bao and D. Jaksch, SIAM (Soc. Ind. Appl. Math.) J. Numer. Anal. 41, 1406 (2004)

${ }^{30}$ P. A. Ruprecht, M. J. Holland, K. Burnett, and M. Edwards, Phys. Rev. A 51, 4704 (1995).

${ }^{31}$ W. H. Press, S. A. Teukolsky, W. T. Vetterling, and B. P. Flannery, Numerical Recipes: The Art of Scientific Computing, 3rd ed. (Cambridge University Press, New York, 2007).

${ }^{32}$ J. M. Soler, E. Artacho, J. D. Gale, A. García, J. Junquera, P. Ordejón, and D. Sánchez-Portal, J. Phys. Condens. Matter 14, 2745 (2002).

${ }^{33}$ X. Qian, J. Li, X. Lin, and S. Yip, Phys. Rev. B 73, 035408 (2006).

${ }^{34}$ W. Magnus, Commun. Pure Appl. Math. 7, 649 (1954).

${ }^{35}$ A. Ojanperä, V. Havu, L. Lehtovaara, and M. Puska, J. Chem. Phys. 136, 144103 (2012)

${ }^{36}$ I. Tavernelli, Phys. Rev. B 73, 094204 (2006).

${ }^{37}$ E. P. Fowe and A. D. Bandrauk, Phys. Rev. A 81, 023411 (2010).

${ }^{38}$ S. Bubin and K. Varga, Phys. Rev. B 85, 205441 (2012).

${ }^{39}$ F. Calvayrac, P.-G. Reinhard, and E. Suraud, J. Phys. B 31, 5023 (1998).

${ }^{40}$ F. Gygi, E. W. Draeger, M. Schulz, B. R. de Supinski, J. A. Gunnels, V. Austel, J. C. Sexton, F. Franchetti, S. Kral, C. W. Ueberhuber, and J. Lorenz, in Proceedings of the 2006 ACM/IEEE Conference on Supercomputing, SC'06 (ACM, New York, 2006).

${ }^{41}$ J. L. Alonso, X. Andrade, P. Echenique, F. Falceto, D. Prada-Gracia, and A. Rubio, Phys. Rev. Lett. 101, 096403 (2008). 